\title{
Phonon coupling and shallow defects in $\mathrm{CuInS}_{2}$
}

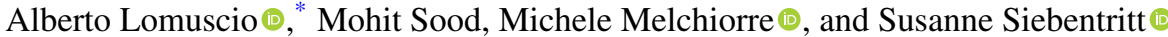 \\ Laboratory for Photovoltaics, Physics and Materials Science Research Unit, University of Luxembourg, \\ 44, rue du Brill, 4422-Belvaux, Luxembourg
}

(Received 2 November 2019; revised manuscript received 30 January 2020; accepted 31 January 2020; published 12 February 2020)

\begin{abstract}
$\mathrm{Cu}(\mathrm{In}, \mathrm{Ga}) \mathrm{S}_{2}$ is an emerging material for solar cells, reaching already efficiencies above $15 \%$. For any solar cell material, it is essential to understand the recombination processes and doping behavior of its defects. Therefore, in this work, we present a detailed photoluminescence and admittance investigation of polycrystalline $\mathrm{CuInS}_{2}$ thin films to study the shallow intrinsic defects. We find evidence for two acceptors, 105 and $145 \mathrm{meV}$ from the valence band, their predominance depending on the $\mathrm{Cu}$ excess, plus one donor, $30 \mathrm{meV}$ from the conduction band. The high crystalline and electronic quality of our samples allows the detection of low intensity luminescence peaks. Based on these emissions we can analyze the phonon coupling of the observed defects and show that an emission previously attributed to a second donor is in fact a phonon replica of the first donor-acceptor transition.
\end{abstract}

DOI: 10.1103/PhysRevB.101.085119

\section{I. $\mathrm{CuInS}_{2}$ FOR TANDEM SOLAR CELLS}

Thin film solar cells present the electricity source with the lowest carbon footprint [1]. Efforts to develop thin film tandem solar cells are motivated by the expected progress in efficiency towards $30 \%$ based on solar cells with a low energy consumption [2]. Thin film solar cells based on the chalcopyrite material $\mathrm{Cu}(\mathrm{In}, \mathrm{Ga})(\mathrm{S}, \mathrm{Se})_{2}$ (CIGSSe) have reached efficiency above 23\% [3]. The material is an alloy of $\mathrm{CuInSe}_{2}$, $\mathrm{CuGaSe}_{2}, \mathrm{CuInS}_{2}$, and $\mathrm{CuGaS}_{2}$. The band gap of the compound increases with $\mathrm{Ga}$ and with $\mathrm{S}$ content. The highest efficiencies are reached with a bandgap of $1.1 \mathrm{eV}$. However, new efforts have been undertaken to boost performance of solar cells with lower band gap [4,5], as they would be suitable as bottom cells in a tandem configuration [2]. Currently, most of the research on tandem solar cells is focused on perovskite cells on top of silicon or CIGSe bottom cell [6-9]. An emerging candidate for top cell is $\mathrm{Cu}(\mathrm{In}, \mathrm{Ga}) \mathrm{S}_{2}$. Its band gap energy can be tuned between $1.55 \mathrm{eV}$ (pure $\mathrm{CuInS}_{2}$ ) and $2.53 \mathrm{eV}$ (pure $\mathrm{CuGaS}_{2}$ ) [10], thus matching well the solar spectrum for energy conversion both for single- and multijunction device [11].

A step forward was recently made for the sulphide chalcopyrite $\mathrm{Cu}(\mathrm{In}, \mathrm{Ga}) \mathrm{S}_{2}$, achieving a record efficiency of $15.5 \%$ [12] and open circuit voltage Voc up to $973 \mathrm{mV}$ [13,14]. Thus, for further improvement of single- and multijunction solar cells it will be essential to understand the electronic properties

\footnotetext{
*Corresponding author: alberto.lomuscio@uni.lu
}

Published by the American Physical Society under the terms of the Creative Commons Attribution 4.0 International license. Further distribution of this work must maintain attribution to the author(s) and the published article's title, journal citation, and DOI. of $\mathrm{CuInS}_{2}$, which is part of the record efficiency alloy and has a great potential in tandem solar cells.

Recently, we showed that a deep defect in $\mathrm{CuInS}_{2}$ with a luminescence around $0.8 \mathrm{eV}$ is a major recombination center. We could demonstrate that the concentration of this defect is reduced with higher deposition temperature and higher $\mathrm{Cu}$ content $[15,16]$.

Here we investigate the spectrum of the shallow defects, which are responsible for the $p$-type doping of the material.

Although less studied than $\mathrm{Cu}(\mathrm{In}, \mathrm{Ga}) \mathrm{Se}_{2}$, there have been several studies, based on photoluminescence (PL), to identify the energy levels of the intrinsic defects in $\mathrm{CuInS}_{2}$ (single crystals and films), focusing mostly on the shallow defects.

The general agreement is that the near band gap luminescence is characterized by the presence of two shallow acceptors and two shallow donors, even if there is no agreement on their activation energies [17-20]. Another common feature reported in low temperature PL spectra is the presence of a broad deep band in the range of $1.2-1.3 \mathrm{eV}$, with an intensity comparable to the near band edge transitions [20,21].

A first step towards identifying the chemical nature of the defects behind the observed energy levels is to study their correlation with composition of the as grown films. However, no systematic compositional study is available and observed differences between the various studies can also be due to different preparation conditions. Therefore, a detailed compositional investigation is needed, analyzing absorbers grown by the same deposition process.

Here we investigate polycrystalline $\mathrm{CuInS}_{2}$ thin films deposited by coevaporation at high temperatures. The indium amount evaporated has been tuned to obtain different compositions in the final absorbers, described by the $\mathrm{Cu} / \mathrm{In}$ ratio. It is worth mentioning for samples grown under $\mathrm{Cu}$ excess, thus $\mathrm{Cu} / \mathrm{In}>1$, the final films include both a stoichiometric chalcopyrite phase and a secondary $\mathrm{CuS}_{x}$ phase [22]. 
We show that at $10 \mathrm{~K}$ the PL spectra show strong excitonic luminescence, indicating the high electronic quality of the films. Additionally, we detect two donor-acceptor (DA) transitions: one around $1.43 \mathrm{eV}$ dominating at low $\mathrm{Cu}$ excess (DA1), and one around $1.39 \mathrm{eV}$, dominating at high $\mathrm{Cu}$ excess (DA2). Their origin is traced to the transition between a common donor and two different acceptors.

The high quality of the absorbers investigated leads to a very low intensity of the deep defect luminescence in the range of $1.2-1.3 \mathrm{eV}$. This feature allows one to doubtlessly attribute the defect related emission at lower energies than the two DA transitions to phonon replicas of those transitions, in contradiction to the models reported in literature. Therefore, there is no evidence for a second deeper donor. Here, we demonstrate the remarkable similarity between the sulphide material and the better investigated selenide material [23].

\section{EXPERIMENTAL DETAILS}

The $\mathrm{CuInS}_{2}$ (CIS) absorbers were deposited on Mo-coated high temperature glass, which allows process temperatures above those used with soda-lime glass. In the present work, thin films were grown by coevaporation of $\mathrm{Cu}$, In, and $\mathrm{S}$ in a physical vapour deposition system using a deposition temperature of $650^{\circ} \mathrm{C}$. To achieve different $\mathrm{Cu} / \mathrm{In}$ ratios for the final samples, the Indium flux has been tuned, while keeping all the other parameters constant. The compositions reported are measured by energy dispersive $\mathrm{x}$-ray spectroscopy (EDX) before etching and represent an average of the chalcopyrite and the $\mathrm{CuS}_{x}$ phase. The secondary phase $\mathrm{CuS}_{x}$ for the $\mathrm{Cu}$-rich samples was removed by potassium cyanide $(\mathrm{KCN})$ etching performed with a $10 \%$ aqueous solution for $5 \mathrm{~min}$. After this cyanide treatment, the $\mathrm{Cu} / \mathrm{In}$ ratio was close to 1 , determined by EDX. In fact, concerning the final compositions of the $\mathrm{Cu}$-rich as grown films, they all reflect a stoichiometric CIS layer together with the $\mathrm{CuS}_{x}$ phase [22]. As the $\mathrm{Cu} / \mathrm{In}$ ratio is tuned above 1 during the depositions, the CIS layers are not grown off-stoichiometric, but the $\mathrm{Cu}$ excess translates into a thicker layer of $\mathrm{CuS}_{x}$ phase.

Low temperature photoluminescence measurements were carried out in a home-built system with a $663-\mathrm{nm}$ wavelength diode laser as excitation source, introducing the samples (after the KCN etching step) into a helium flow cryostat. The emitted luminescence was collected by two off-axis parabolic mirrors, focused into a fiber, spectrally resolved by a monochromator and detected by a Si-charge-coupled device (CCD) and an InGaAs diode array. The setup allows a wavelength resolution in the visible of about $2 \mathrm{~nm}$. The raw data are corrected for the spectral response of the system using a commercial calibration lamp with a known spectrum. For these measurements, a focusing convex lens has been used, that leads a spot size radius of about $90 \mu \mathrm{m}$, measured by a CCD camera. For excitation intensity dependent PL, the laser power has been attenuated by a filter wheel with neutral density filters.

Admittance measurements have been performed on absorbers with Schottky contact, i.e., Mo/CuInS $2 / \mathrm{Al}$ and absorbers with a buffer-window stack, i.e., $\mathrm{Mo} / \mathrm{CuInS}_{2} / \mathrm{ZnOS} /$ AZO/Ni-Al complete solar cell. The samples were mounted inside a closed-cycle cryostat setup on top of a cold finger. The

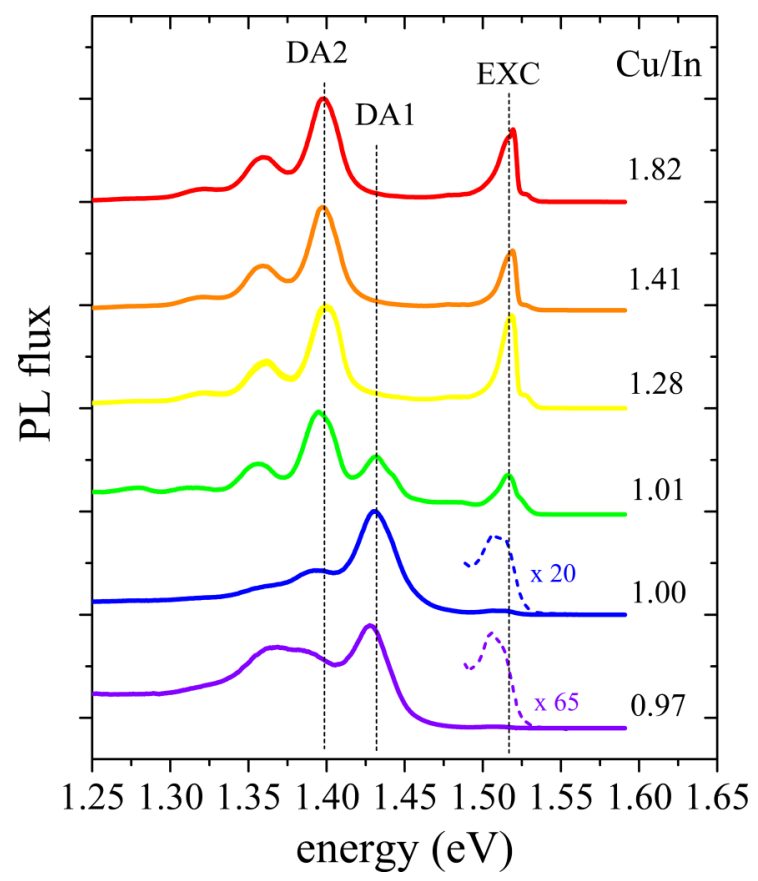

FIG. 1. Photoluminescence spectra of $\mathrm{CuInS}_{2}$ with different compositions measured at $10 \mathrm{~K}$. The excitonic luminescence for the two samples with the lowest $\mathrm{Cu} / \mathrm{In}$ ratio is magnified for a better visibility.

admittance of the sample was recorded in the frequency range of $20 \mathrm{~Hz}$ to $2 \mathrm{MHz}$ with a small signal AC pulse of $30-\mathrm{mV}$ rms. The sample admittance was measured in the temperature range of 320 to $45 \mathrm{~K}$ sample temperature. In order to correctly measure the sample temperature a Si-diode sensor was glued onto an identical glass substrate, which was then placed next to the sample.

\section{NEAR EDGE PHOTOLUMINESCENCE OF CIS}

We investigate the effect of the composition of $\mathrm{CuInS}_{2}$ thin films on the shallow defect structure. Figure 1 gives an overview of the PL spectra for absorber layers with varying $\mathrm{Cu} / \mathrm{In}$. Note, that this is the overall composition of the sample, including the chalcopyrite and the $\mathrm{Cu}_{2} \mathrm{~S}$ secondary phase, which forms for $\mathrm{Cu}$-rich compositions [22], whereas the PL measurements have been performed after KCN etching, which selective removes the secondary phase [24]. After the KCN etching the $\mathrm{Cu} / \mathrm{In}$ ratio is 1 (measured by EDX): therefore, any features observed and discussed in the following are characteristic of the chalcopyrite phase. We observe the following trend: at high $\mathrm{Cu}$ excess we see strong band edge emissions around $1.52 \mathrm{eV}$ together with a peak at $1.39 \mathrm{eV}$ followed by other peaks at lower energies with lower intensities; for low $\mathrm{Cu}$ - excess, the transition at $1.43 \mathrm{eV}$ appears and becomes the dominant one and the band edge emission is much lower in intensity.

\section{A. Band edge emissions}

The band edge emission will be discussed using the data of the sample with $\mathrm{Cu} / \mathrm{In}$ ratio of 1.8 as it shows the highest intensity and the best-resolved emissions. This suggests an 


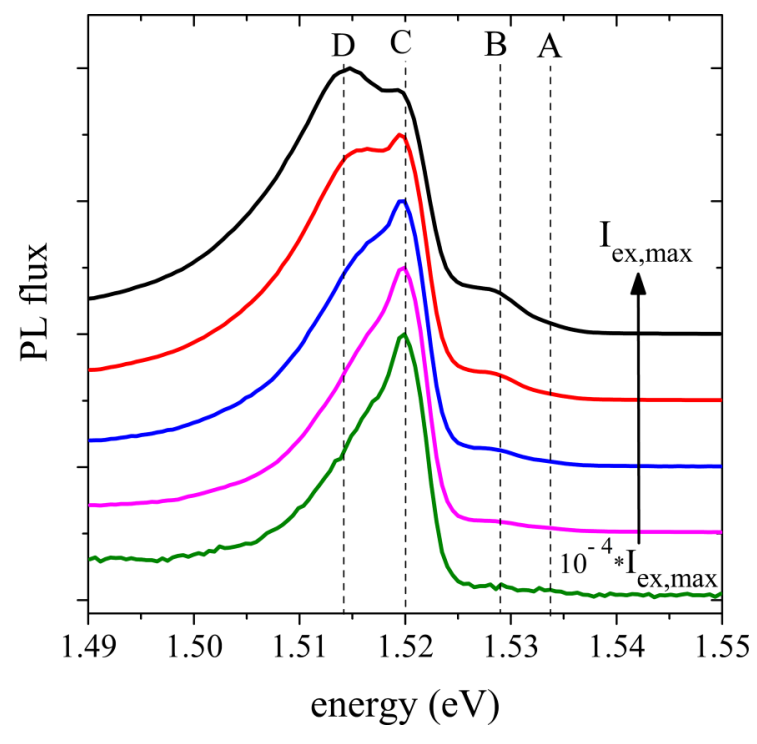

FIG. 2. Intensity dependence of the band edge photoluminescence spectra measured at $10 \mathrm{~K}$ on the sample with a $\mathrm{Cu} / \mathrm{In} 1.8$. The spectra have been normalized and shifted in intensity for a better visualization. The vertical lines mark the energies of the different emissions.

improvement in the crystalline quality of the stoichiometric chalcopyrite phase when films are grown under Cu excess, as already found in our previous work [15]. In Fig. 2 we present those emissions measured at different excitation intensity.

The first clear feature is that they do not show any peak shift with different excitations over four orders of magnitude, which means they cannot be donor-acceptor transitions [25]. At these low temperature they are either excitonic lines or free-to-bound (FB) transitions of shallow defects [25]. Four different transitions are identified, at $1.514 \mathrm{eV}(D), 1.520 \mathrm{eV}$ $(C), 1.528 \mathrm{eV}(B)$, and $1.534 \mathrm{eV}(A)$. In Fig. 3(a) we report the PL flux $\left(I_{\mathrm{PL}}\right)$ of these transitions as a function of the excitation intensity $\left(I_{\mathrm{ex}}\right)$ in a double logarithmic plot.

Excitons should follow a power law as $I_{\mathrm{PL}} \sim\left(I_{\mathrm{ex}}\right)^{k}$ with a super-linear behavior [26], i.e., with $k>1$. The corresponding exponents for each transition, determined over more than four order of magnitude in excitation intensity, are reported in Fig. 3.

All exponents are close to $3 / 2$. The emission $D(1.514 \mathrm{eV})$, $B(1.528 \mathrm{eV})$, and $A(1.534 \mathrm{eV})$ are well described by a single power law. The $C$ line $(1.520 \mathrm{eV})$, however, does not show a straight line in the log-log plot, although it can be fitted by an exponent of 1.45. Figure 3(b), in fact, shows its trend might be well fitted as a combination of two power laws, with exponents of $3 / 2$ and $2 / 2$, respectively. The fit equation with transitions between different exponents is given in the Supplemental Material [27]. Indeed, dependences more complex than single power law have been reported for excitation intensity photoluminescence $[26,28]$ : based on the rate equations of different processes, it becomes obvious that the exponent $k$ takes on only fixed values of multiples of $1 / 2$ at low temperature, where only photoexcited carriers are available. The differences are due to the availability of competing recombination channels (even through deep defects). The change of exponent for different excitation occurs when
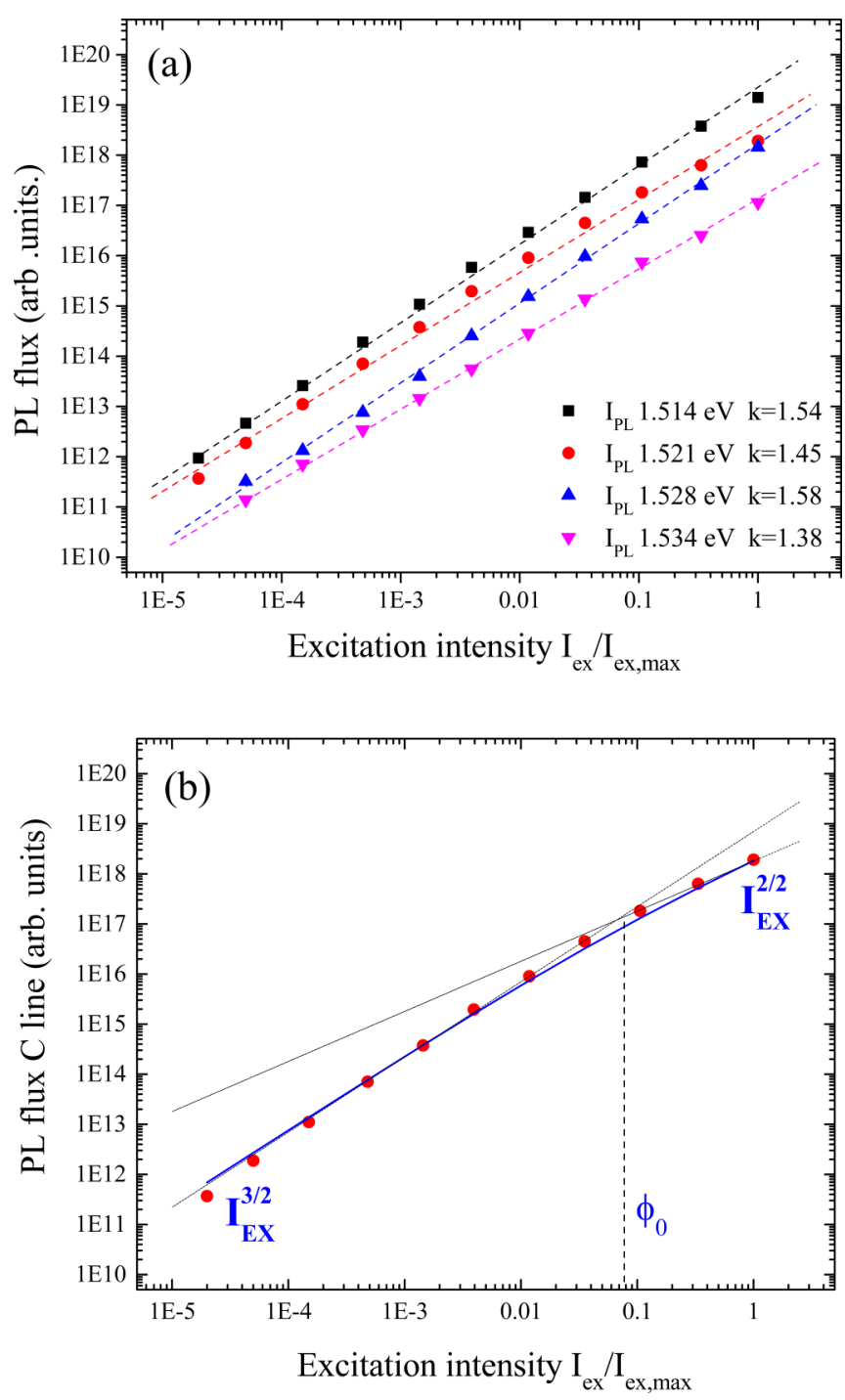

FIG. 3. (a) Excitation intensity dependence of the integrated PL flux of the four band edge emissions taken from the sample with a $\mathrm{Cu} / \mathrm{In}$ ratio of 1.8 . (b) Trend of the $1.520 \mathrm{eV}$ emission (line C) together with 2 power laws with a $k$ value of $3 / 2$ and $2 / 2$, respectively. $\Phi_{0}$ indicates the excitation threshold.

one of the defects involved in the transition or in one of the competing transitions saturates. This leads to a curved dependence in the log-log plot, which can be fitted in general with exponents different from $\mathrm{n} / 2$ : the latter case occurs predominantly in transitions regions between two limiting cases of a single power law. The behavior measured for the line $C$ is best explained by a FB transition, where the shallow defect saturates [28]. A more detailed explanation is reported in the Supplemental Material.

The $A$ line $(1.534 \mathrm{eV})$ has been attributed previously to the recombination of free exciton [29]. The exciton ionization energy $\left(E_{X}\right)$ of about $20 \mathrm{meV}$ has been determined in the past both by the analysis of the quenching of the emission intensity [29] and by comparison of the first and second excited states of the free exciton [30]. Adding this ionization energy to the emission energy of the free exciton, a band gap of $1.554 \mathrm{eV}$ at $10 \mathrm{~K}$ can be determined. This value matches well those reported in the literature [29-31]. 
TABLE I. Possible values of donor and acceptor activation energies (in meV) calculated using Eqs. (1)-(4) for the emission lines $B$ and $D$. The errors reported have been estimated assuming an uncertainty of $\pm 2 \mathrm{meV}$ of the band gap value.

\begin{tabular}{lcccrr}
\hline \hline Emission line & Emission energy $(\mathrm{eV})$ & Neutral donor & Ionized donor & Neutral acceptor & Ionized acceptor \\
\hline$B$ & 1.528 & $22 \pm 7$ & $25 \pm 4$ & $100 \pm 30$ & $18 \pm 2$ \\
$D$ & 1.514 & $74 \pm 7$ & $38 \pm 4$ & $335 \pm 30$ & $26 \pm 2$ \\
\hline \hline
\end{tabular}

We attribute the other two lines $B$ and $D$ to bound excitons, in agreement with the literature [29]. By using Hayne's rule, it is possible to correlate the bound exciton emission energies to the ionization energy of the corresponding donor or acceptor [32-36]. In particular, Binsma et al. [29], using an electron effective mass $m_{e}=0.16$ and hole effective mass $m_{p}=1.6$, estimated the exciton-neutral donor $\left(E_{D^{0} X}\right)$ and the excitonneutral acceptor $\left(E_{A^{0} X}\right)$ binding energies to be, respectively,

$$
\begin{aligned}
& E_{D^{0} X}=0.27 E_{D}+E_{X}, \\
& E_{A^{0} X}=0.06 E_{A}+E_{X},
\end{aligned}
$$

whereas for excitons bound to ionized donor $\left(E_{D^{+} X}\right)$ or to ionized acceptor $\left(E_{A^{-} X}\right)$, the binding energies are given by

$$
\begin{gathered}
E_{D^{+} X}=1.044 E_{D}, \\
E_{A^{-} X}=1.5 E_{A} .
\end{gathered}
$$

Thus, the photon energy of an emission line related to a bound exciton is due to the difference between the band gap and the binding energy of the corresponding bound exciton. Therefore, from the exciton emission lines observed experimentally, together with the band gap estimated $(1.554 \mathrm{eV})$, it is possible to calculate the activation energies of the donors or acceptors involved. All calculation are summarized in Table I.

The emissions at 1.514 and $1.528 \mathrm{eV}$ have been reported as bound exciton to neutral donor at about $72 \mathrm{meV}$ and to neutral donor at about $35 \mathrm{meV}$, respectively [29], in good agreement with our results. Nevertheless, it cannot be excluded that the emission line $B$ belongs to a neutral acceptor with an energy of about $100 \mathrm{meV}$ (as it will be discussed in the following) and the emission line $D$ to an ionized donor with an energy of $38 \mathrm{meV}$.

Finally, the $C$ line at $1.520 \mathrm{eV}$ is attributed to the FB transition of a shallow defect, with an energy defect of $34 \mathrm{meV}$, derived as difference between the band gap energy and peak energy line. This value has been attributed to a donor in the literature [29]. The activation energy of the donor will be further discussed in the following section.

Thus from the near band edge emissions, we have correlated two lines to bound excitons ( $B$ and $D$ ), one to free excitonic emission $(A)$, which leads to an estimation of the band gap of $1.554 \mathrm{eV}$ at $10 \mathrm{~K}$, and the last one $(C)$ to a $\mathrm{FB}$ emission, that belongs to a donor defect with an energy about $34 \mathrm{meV}$.

It is worth mentioning that similar transitions have been found for all samples grown with different compositions, shown in Fig. 1. The samples with the lowest $\mathrm{Cu} / \mathrm{In}$ ratios (1.00 and 0.97) show the same lines but with a shift to lower energies. This observation is explained by a lower band gap of the corresponding samples. In Fig. S1 we show the comparison of the band gap luminescence of the samples with $\mathrm{Cu} / \mathrm{In} 1.82$ and 1.00 measured at different temperatures. The latter always shows a shift to lower energy of $8 \mathrm{meV}$, thus indicating a corresponding band gap of $1.546 \mathrm{eV}$ at $10 \mathrm{~K}$. A similar trend has been reported for CISe absorbers [37], where those grown in $\mathrm{Cu}$-poor conditions exhibit lower band gaps then those deposited under $\mathrm{Cu}$ excess.

\section{B. Shallow defects}

In the following, the two main transitions shown in Fig. 1 will be discussed: at the one hand the $1.43-\mathrm{eV}$ transition, characteristic feature of absorbers with a $\mathrm{Cu} / \mathrm{In}$ ratio close to 1 , that will be called DA1, on the other hand the $1.39-\mathrm{eV}$ transition, dominant for absorbers grown under high $\mathrm{Cu}$ excess, which will be called DA2. The PL investigation of the DA1 and DA2 transitions have been performed on the samples with a $\mathrm{Cu} / \mathrm{In}$ ratio of 1.00 and 1.82 , respectively. These main peaks are accompanied by several peaks of decreasing intensity at lower energies. Before discussing the two main transitions in detail, we first analyze these additional emissions.

\section{Phonon coupling}

In Fig. 4, the low temperature PL spectra are shown for the DA1 and the DA2 transitions. It is clear that in both cases the main peak is followed by other transitions at lower energies with lower intensities. We attribute these emissions to phonon replicas. Three arguments support this attribution: (i) the spectral spacing between two consecutive lines is about $39 \mathrm{meV}$, the energy of one of the LO phonons of $\mathrm{CuInS}_{2}$ $[38,39]$; (ii) the trend of the low energy peaks follows the same of the main peak, as a function of the excitation intensity and temperature (Fig. S2 in the Supplemental Material); (iii) they can be well fitted by a Huang-Rhys model, which describes the spectrum as a Poisson distribution of phonon replica according to [40]

$$
I \propto \sum_{n} e^{-S} \frac{S^{n}}{n !},
$$

with $n$ being the number of phonons involved in the transition with intensity $I_{n}$ and $S$ the Huang-Rhys factor, which describes the strength of the electron-phonon coupling in this particular defect. In particular, an $S$ value of about $0.22 \pm 0.03$ has been determined from the fit of the DA1 transition [Fig. 4(a)], and $S=0.48 \pm 0.03$ for the DA2 [Fig. 4(b)]. This trend of $S$ is expected, since a deeper defect should show a stronger electron- phonon coupling, because it is more localized [25].

As it is possible to see from Fig. 4, a third deeper band centered at about $1.25 \mathrm{eV}$ is needed to well fit the low energy side of the PL spectra. A very similar emission has been reported in literature. In those previous studies the intensity 

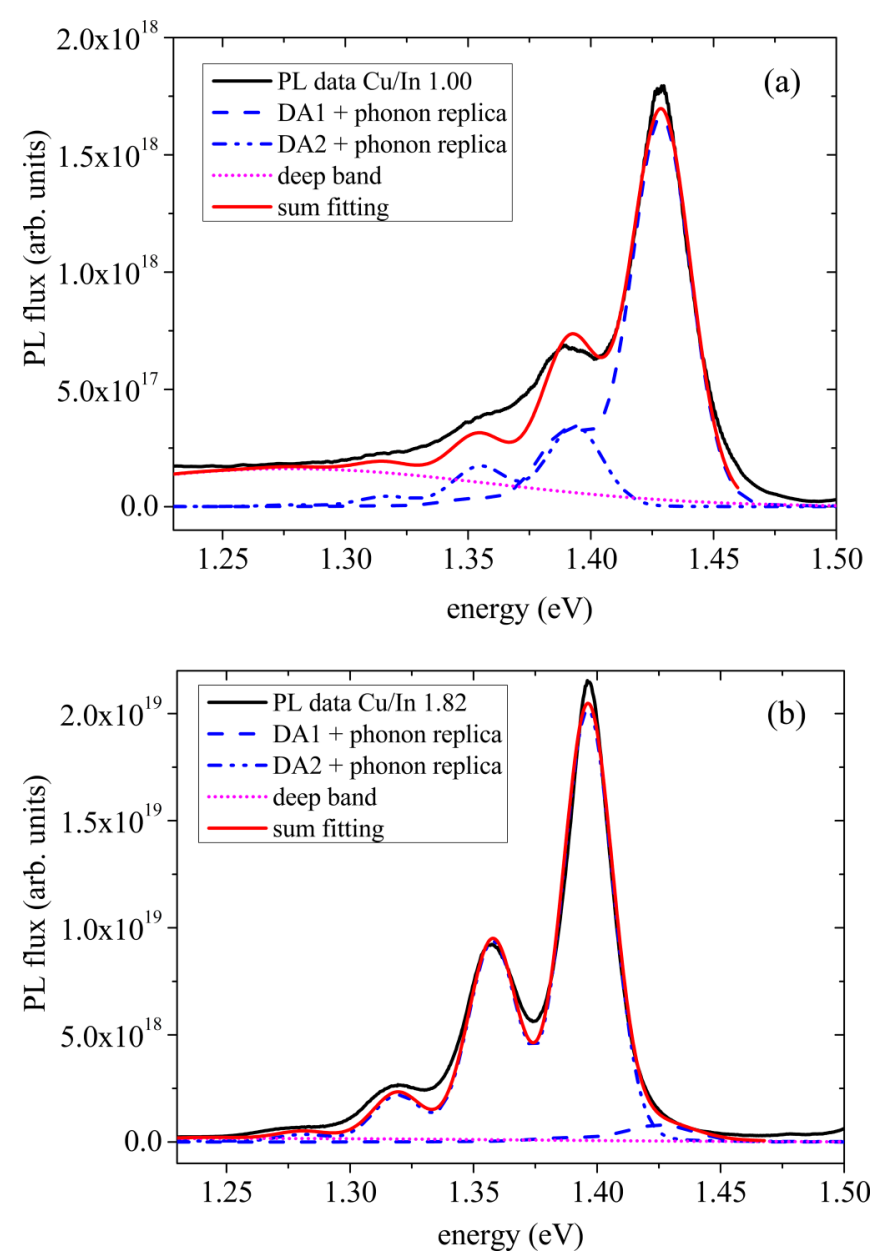

FIG. 4. Measured photoluminescence spectra at $10 \mathrm{~K}$ (black) of $\mathrm{CuInS}_{2}$ thin film with $\mathrm{Cu} / \mathrm{In}$ (a) 1.00 and (b) 1.83 . Both spectra are fitted reasonably well with a Huang-Rhys spectrum of the DA1 and the DA2 plus a contribution of an additional deep emission (purple dotted line).

of this emission was comparable to the one of the shallow transitions [20,21]. This high intensity of the deep emission hid the phonon replicas with $n>1$.

Therefore, all four peaks clearly visible in the energy range near DA1 and DA2 are thus attributed in each case to a single DA transition with its phonon replicas. Thus, the only transitions remaining to be discussed are the main ones, DA1 and DA2.

\section{Excitation and temperature dependence of DA1 and DA2}

Excitation intensity dependence of the energy positions for the peak DA1 and DA2 are reported in Fig. 5: both peaks shift to higher energies with excitation.

The blue shift of the peak position as a function of the excitation intensity is a mark of the donor-to-acceptor nature of these transitions: in fact, for this kind of emissions, the peak energy position is given by [25]

$$
E_{\mathrm{PL}} \propto E_{G}-\left(E_{A}+E_{D}\right)+\frac{e^{2}}{4 \pi \varepsilon_{0} \varepsilon_{r} r},
$$
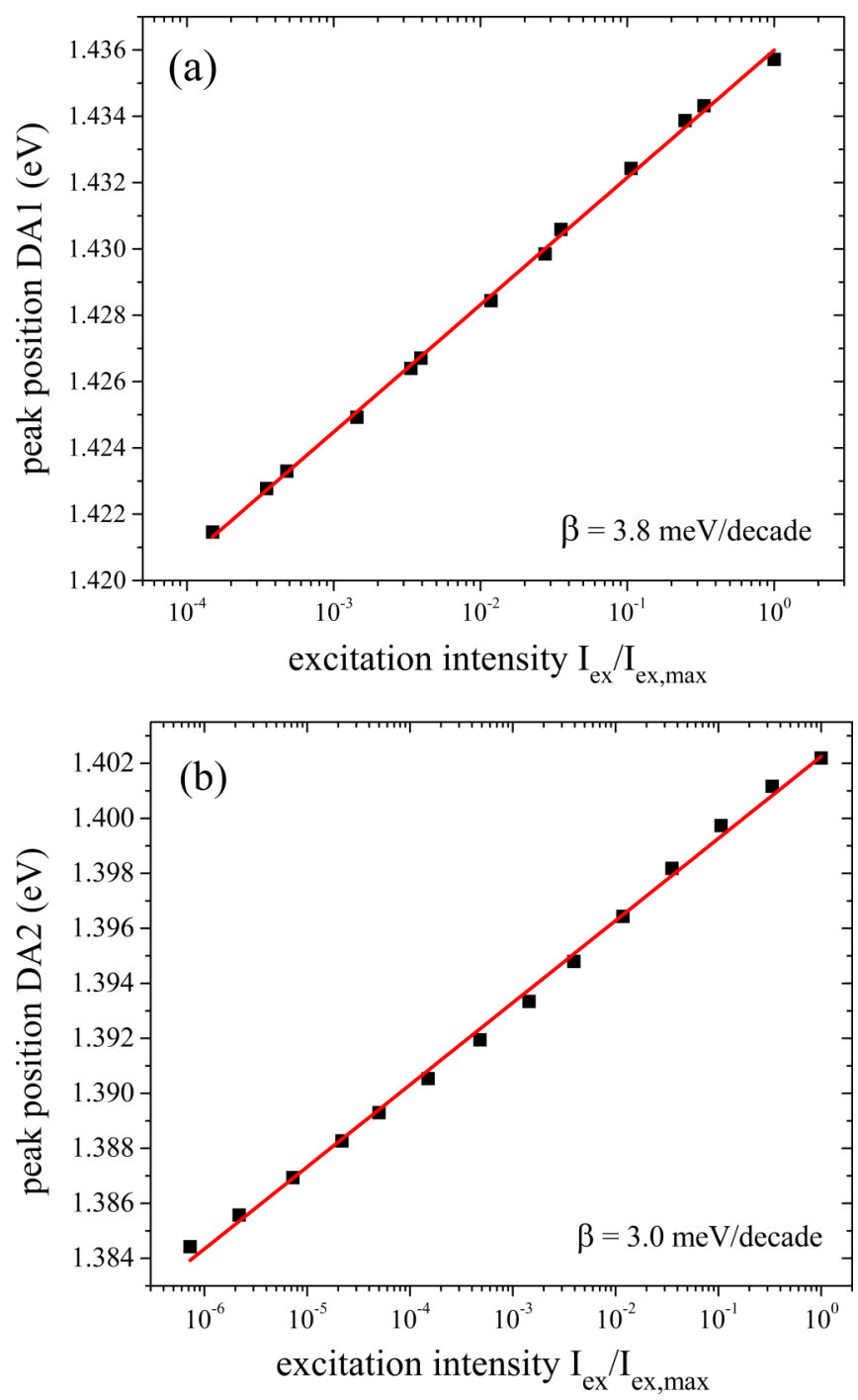

FIG. 5. Excitation intensity dependent energy position of the two DA transitions in $\mathrm{CuInS}_{2}$ taken from the spectra of the samples with $\mathrm{Cu} / \mathrm{In}$ ratio of (a) 1.00 and (b) 1.82 .

with $E_{\mathrm{PL}}$ peak energy position, $E_{G}$ band gap, $E_{A}$ and $E_{D}$ donor and acceptor defect energy and the last term of the equation given by the Coulomb energy, with $e$ elementary charge, $\varepsilon_{0}$ vacuum permittivity, $\varepsilon_{r}$ relative permittivity of the semiconductor material and $r$ distance between donor and acceptor. With increasing excitation, the density of defects involved in the emission increases, thus reducing the average distance $r$ and leading to a blue shift of $E_{\mathrm{PL}}$. This blue shift as a function of excitation intensity can be described by the empirical equation [25]

$$
E_{\mathrm{PL}}\left(I_{\mathrm{ex}}\right)=E_{\mathrm{PL}}\left(I_{\mathrm{ex}, \max }\right)+\beta \log _{10}\left(\frac{I_{\mathrm{ex}}}{I_{\mathrm{ex}, \max }}\right) .
$$

For the above-mentioned peaks, a $\beta$ factor of 3.8 and $3.0 \mathrm{meV}$ per decade have been determined for DA1 and DA2, respectively, which is typical for donor-acceptor transitions in weakly compensated semiconductors [41].

In Fig. 6 we report the PL fluxes $\left(I_{\mathrm{PL}}\right)$ of these transitions as a function of the excitation intensity $\left(I_{\mathrm{ex}}\right)$ in a double 

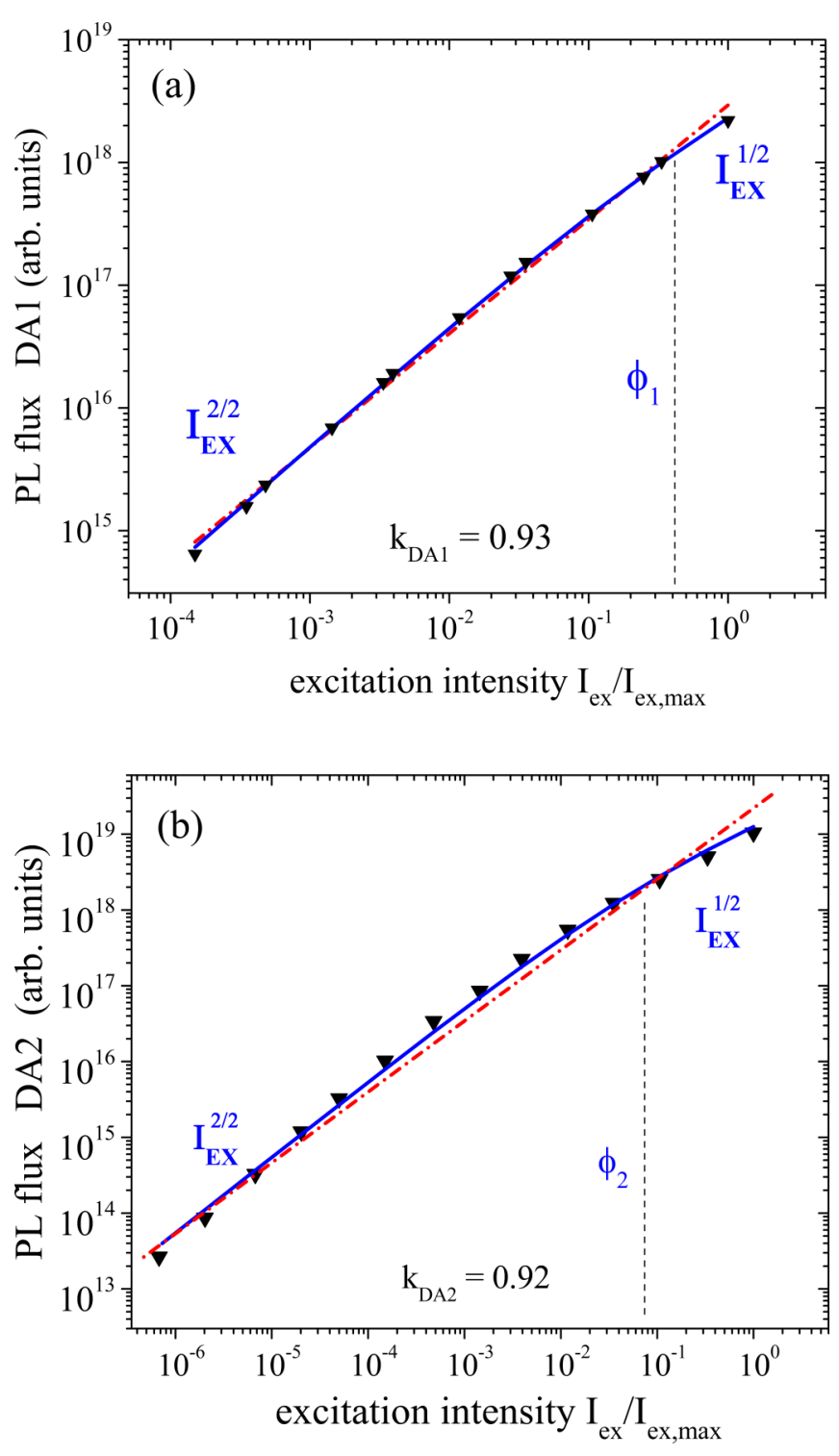

FIG. 6. Excitation intensity dependent intensity of the two DA transitions in $\mathrm{CuInS}_{2}$ taken from the spectra of the samples with $\mathrm{Cu} / \mathrm{In}$ ratio of (a) 1.00 and (b) 1.82 . The dash-dotted lines represent the single power law, while the line of multiple power laws, with the corresponding $k$ values, are reported as well. $\Phi_{1}$ and $\Phi_{2}$ indicate the excitation thresholds.

logarithmic scale. The fitting performed using a single power law leads to a $k$ value of 0.93 and 0.92 for DA1 and DA2, respectively. Nevertheless, as mentioned before in the band edge section, the $k$ value at low temperatures can take on only fixed values of multiples of $1 / 2$, and values different from $n / 2$ are just due to transitions regions between two limiting cases. Indeed, in both DA1 and DA2 it is shown that the measured trend can be fitted as a combination of multiple power laws. While the data of DA1 is compatible with an exponent close to 1 , the log-log plot of DA2 is clearly curved, changing the exponent from 1 to $1 / 2$. This happens when one of the defects involved in the DA transition saturates. It is worth noting that the excitation threshold $\Phi_{2}$ for the DA2 transition occurs in the same range $\left(\sim 8 \times 10^{-2}\right)$ of $\Phi_{0}$, found previously for the FB transition $[C$ line, Fig. 3(b)]. This can be explained assuming that the defect responsible for the FB line at $1.520 \mathrm{eV}$ is involved as well in the DA2 transition; the saturation of this defect leads to the curved log-log plot.

To determine the defects energies involved in DA1 and DA2 transitions, temperature dependent measurements have been performed. Ideally, with increasing temperature the shallower of the two defects involved in a DA transition is thermally emptied, leaving a FB emission. In the latter case, the defect energy can be easily measured by the peak position as the Coulomb energy term in Eq. (2) is not present anymore. Therefore, in case of FB transitions, the peak position does not depend anymore on the excitation intensity: this feature can be used to prove the transition from DA to FB emission.

Figure 7 reports the excitation intensity dependence of the energy of the two main transitions, at 80 and $90 \mathrm{~K}$, respectively. As can be seen, both do not shift with excitation intensity (over three orders of magnitude), which means the transition has become a FB emission at these temperatures and the shallower defect is thermally emptied: FB1 at $1.444 \mathrm{eV}$ and FB2 at $1.411 \mathrm{eV}$. FB2 is observed in samples with a high $\mathrm{Cu} / \mathrm{In}$ ratio, in which from the free exciton emission position at $90 \mathrm{~K}$, we determined a bandgap of $1.556 \mathrm{eV}$. The energy difference between band gap and FB emission gives directly the defect energy of $145 \pm 5 \mathrm{meV}$. As discussed above, the band gap of the sample that shows the FB1 transition (low $\mathrm{Cu} / \mathrm{In}$ ) has a lower band gap by $8 \mathrm{meV}$, which means at $80 \mathrm{~K}$ a value of about $1.548 \mathrm{eV}$ : this allows the determination of the energy of the defect involved in FB1, namely $104 \pm 5 \mathrm{meV}$. The presence of an acceptor with an activation energy of about $105 \mathrm{meV}$ confirms that the attribution of the excitonic $B$ line is not straightforward, and it can be thus related to either a donor or an acceptor, as reported in Table I. At the moment, with the present data, the assignment cannot be discerned.

The presence of a defect of $145 \mathrm{meV}$ in absorbers grown in $\mathrm{Cu}$-rich conditions has been confirmed by admittance measurements performed both on Mo/CuInS $2 / \mathrm{Al}$ (Fig. 8) and on $\mathrm{Mo} / \mathrm{CuInS}_{2} / \mathrm{ZnOS} / \mathrm{ZnO}: \mathrm{Al} / \mathrm{Al}$ stacks (Fig. S3). In both cases, a capacitance step has been found in the range 100-180 K with activation energies $140-145 \mathrm{meV}$, that well match the one found by PL investigation. It is worth mentioning also for admittance spectroscopy the CIS films have been KCN-etched to remove any secondary phases prior the deposition of additional layers needed to carry out the measurements. Taking into account the accuracy of temperature and frequency measurements, an error of about $\pm 5 \mathrm{meV}$ of the activation energy of the capacitance step is derived. To investigate the origin of this capacitance step, we measured the activation energy of series resistance and diode current at a fixed high forward bias as a function of temperature. Both give an activation energy value well below $142 \mathrm{meV}$, indicating that this last step originates from a defect and not from a barrier. Additionally, similar measurements have been performed both with reverse and forward DC bias, without any significant changes of the main capacitance activation energy $(140 \mathrm{meV})$ within error. With these observations, the origin as interface defect can be ruled out, as in this case the activation energy would be bias dependent.

With the two defect activation energies determined (responsible for FB1 and FB2 emissions), together with the peak positions of DA1 and DA2 transitions shown in Fig. 5, 

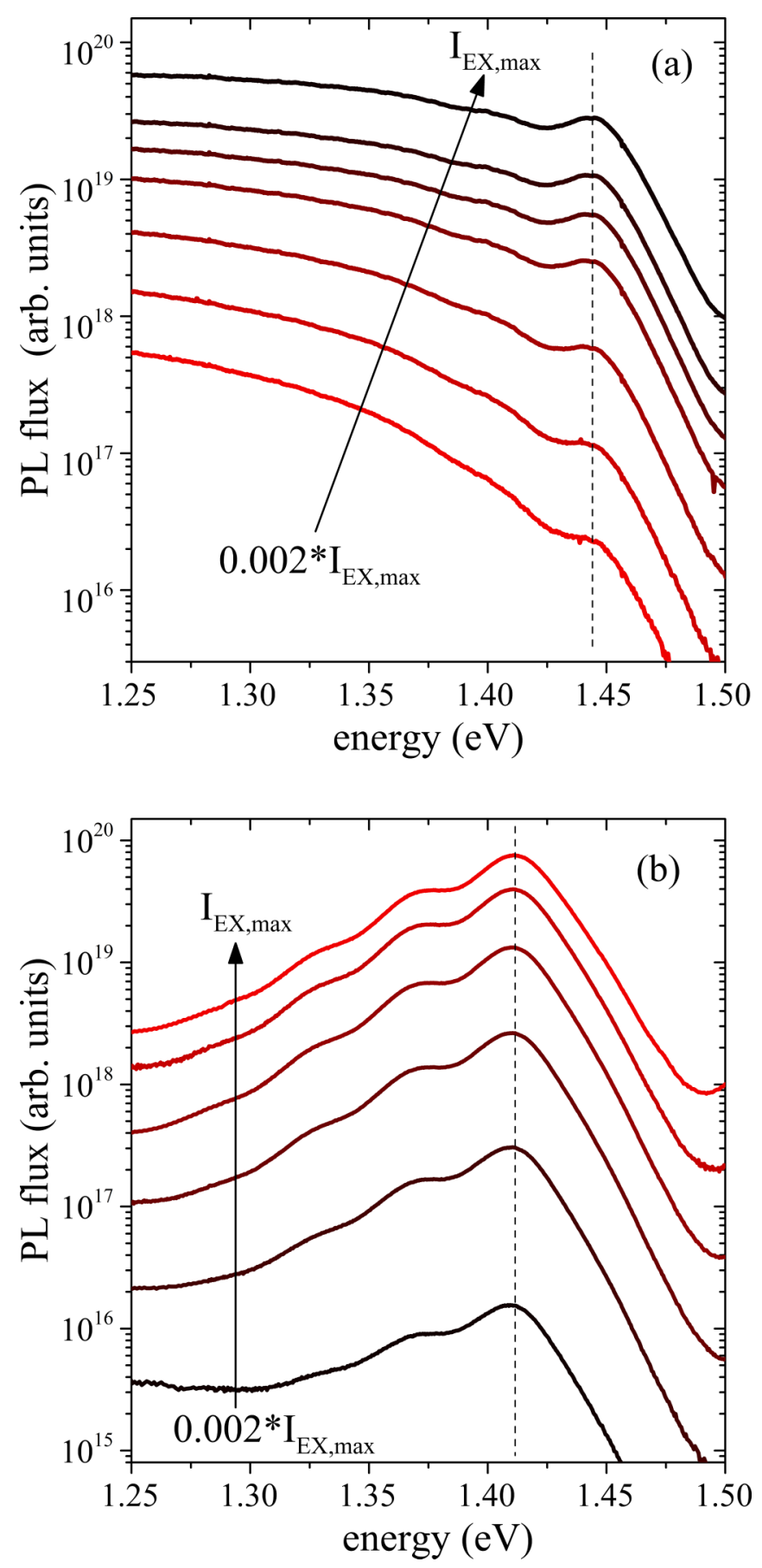

FIG. 7. Excitation intensity dependent spectra of the two FB transitions in $\mathrm{CuInS}_{2}$ taken from the spectra with $\mathrm{Cu} / \mathrm{In}$ ratio of (a) 1.00 for FB1 and (b) 1.82 for FB2.

we can verify the activation energy of the shallower defect involved in both transitions. As discussed in Eq. (6), the peak position of a DA transition shifts due to the Coulomb energy contribution. For the peak positions measured at the lowest excitation intensity, this contribution is expected to be almost negligible. For the DA1 transition, the peak position measured at the lowest excitation intensity is at $1.421 \mathrm{eV}$ : the deeper defect involved has an energy of $104 \mathrm{meV}$, then the shallower defect activation energy can be estimated at about $21 \mathrm{meV}$. From the DA2 transition, the estimated activation

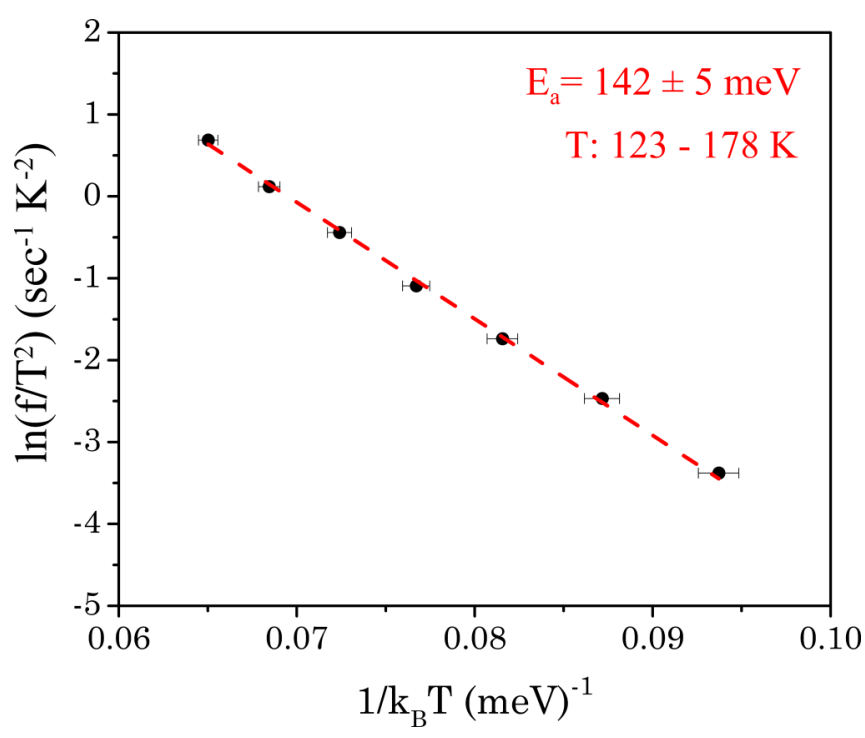

FIG. 8. Arrhenius plot for $\mathrm{Mo} / \mathrm{CuInS}_{2} / \mathrm{Al}$ stack indicating the activation energy of the main capacitance step for the CIS samples grown in $\mathrm{Cu}$-rich excess.

energy of the shallower defect is about $25 \mathrm{meV}$. From the FB transition observed at low temperatures (line $C$ of the band edge emission), we extracted a defect energy of $34 \mathrm{meV}$. We postulate that all three transitions (DA1, DA2, and FB) involve the same shallow defect at $30 \pm 10 \mathrm{meV}$.

\section{A DEFECT MODEL FOR CuInS 2}

In $\mathrm{CuInS}_{2}$ absorber layers two main donor-acceptor transitions have been observed in literature, at 1.39 and $1.43 \mathrm{eV}$, respectively. They have been explained by the presence of one common donor and two shallow acceptors. Moreover, both transitions always manifest together with additional peaks at lower energy: these peaks have been interpreted as additional DA transitions involving a deeper donor. Nevertheless,

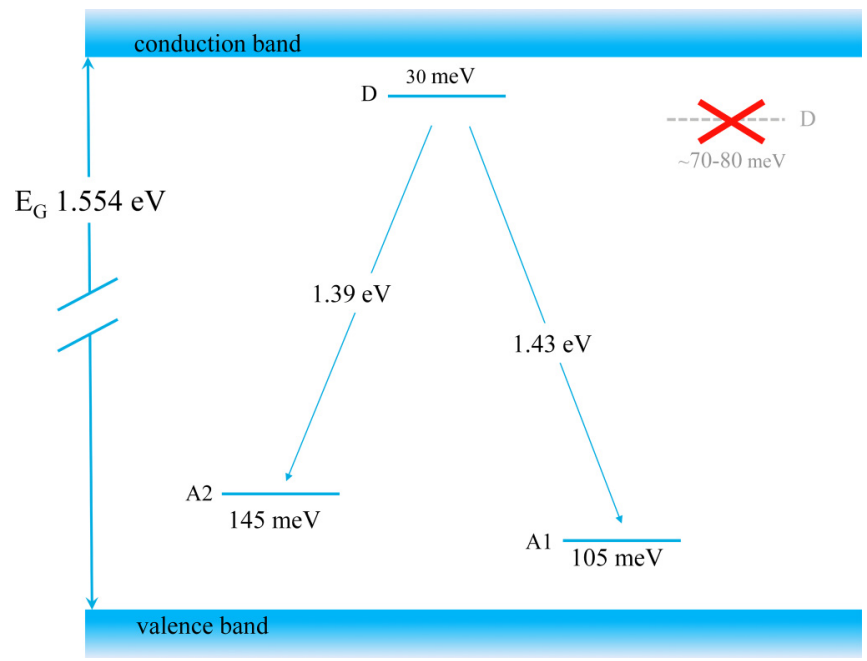

FIG. 9. Recombination model for $\mathrm{CuInS}_{2}$ based on PL measurements. The defect energies reported have to be assumed with an error of about $\pm 5 \mathrm{meV}$. 
there has been no general agreement on their energies (e.g., acceptor activation energies range from 65 to $150 \mathrm{meV}$ ) [17-20]. Here we show by a detailed PL investigation of high quality films that there is no need of a second deeper donor to justify the presence of peaks at lower energies: they can be well fitted by a Huang-Rhys model, therefore they are to be considered phonon replicas of the main transitions. This attribution is possible because of the high quality of the films investigated, that leads to the presence of several phonon replica, whereas in previous literature reports only one phonon replica was observed and has been explained by the presence of a deeper donor [17,20]. By temperature and excitation intensity dependence we demonstrate that the two main transitions become FB emissions in the range of $80-90 \mathrm{~K}$ and from their peak position we have determined the corresponding defect energies reported in the recombination model proposed in Fig. 9. Therefore, these results show there is a remarkable similarity between the sulphide material and the better investigated selenide material [23].

\section{ACKNOWLEDGMENTS}

The authors gratefully acknowledge the Luxembourgish Fonds National de la Recherche (FNR) for funding under the CORRKEST and MASSENA project.

The authors declare no competing financial interests.
[1] E. Hertwich, J. A. de Larderel, A. Arvesen, P. Bayer, J. Bergesen, E. Bouman, T. Gibon, G. Heath, C. Peña, and P. Purohit, Green energy choices: The benefits, risks, and trade-offs of low-carbon technologies for electricity production, United Nations Environment programme (2016), pp. 425-451.

[2] S. Siebentritt, High voltage, please! Nat. Energy 2, 840 (2017).

[3] M. Nakamura, K. Yamaguchi, Y. Kimoto, Y. Yasaki, T. Kato, and $\mathrm{H}$. Sugimoto, $\mathrm{Cd}$-free $\mathrm{Cu}(\mathrm{In}, \mathrm{Ga})(\mathrm{Se}, \mathrm{S})_{2}$ thin-film solar cell with a new world record efficiency of $23.35 \%$, IEEE Journal of Photovoltaics 9, 1863 (2019).

[4] H. Elanzeery, F. Babbe, M. Melchiorre, F. Werner, and S. Siebentritt, High-performance low bandgap thin film solar cells for tandem applications, Prog. Photovolt. Res. Appl. 26, 437 (2018).

[5] T. Feurer, F. Fu, T. P. Weiss, E. Avancini, J. Löckinger, S. Buecheler, and A. Tiwari, RbF post deposition treatment for narrow bandgap $\mathrm{Cu}(\mathrm{In}, \mathrm{Ga}) \mathrm{Se}_{2}$ solar cells, Thin Solid Films 670 , 34 (2019).

[6] https://www.oxfordpv.com/news/oxford-pv-perovskite-solarcell-achieves-28-efficiency.

[7] https://www.imec-int.com/en/articles/perovskite-cigs-tandemcell-with-record-efficiency-of-24-6-percent.

[8] H. Shen, J. Peng, D. Jacobs, N. Wu, J. Gong, Y. Wu, S. K. Karuturi, X. Fu, K. Weber, and X. Xiao, Mechanically-stacked perovskite/CIGS tandem solar cells with efficiency of $23.9 \%$ and reduced oxygen sensitivity, Energy Environ. Sci. 11, 394 (2018).

[9] C. O. R. Quiroz, Y. Shen, M. Salvador, K. Forberich, N. Schrenker, G. D. Spyropoulos, T. Heumüller, B. Wilkinson, T. Kirchartz, and E. Spiecker, Balancing electrical and optical losses for efficient 4-terminal $\mathrm{Si}$-perovskite solar cells with solution processed percolation electrodes, J. Mater. Chem. A 6, 3583 (2018).

[10] B. Tell, J. Shay, and H. Kasper, Electrical properties, optical properties, and band structure of $\mathrm{CuGaS}_{2}$ and $\mathrm{CuInS}_{2}$, Phys. Rev. B 4, 2463 (1971).

[11] S. Siebentritt, What limits the efficiency of chalcopyrite solar cells? Sol. Energy Mater. Sol. Cells 95, 1471 (2011).

[12] H. Hiroi, Y. Iwata, S. Adachi, H. Sugimoto, and A. Yamada, New world-record efficiency for pure-sulfide $\mathrm{Cu}(\mathrm{In}, \mathrm{Ga}) \mathrm{S}_{2}$ thin-Film solar cell with $\mathrm{Cd}$-free buffer layer via $\mathrm{KCN}$-free process, IEEE J. Photovolt. 6, 760 (2016).

[13] H. Hiroi, Y. Iwata, K. Horiguchi, and H. Sugimoto, 960-mV open-circuit voltage chalcopyrite solar cell, IEEE J. Photovolt. 6, 309 (2016).
[14] H. Hiroi, Y. Iwata, H. Sugimoto, and A. Yamada, Progress toward $1000-\mathrm{mV}$ open-circuit voltage on chalcopyrite solar cells, IEEE J. Photovolt. 6, 1630, (2016).

[15] A. Lomuscio, T. Rödel, T. Schwarz, B. Gault, M. Melchiorre, D. Raabe, and S. Siebentritt, Quasi-fermi-level splitting of $\mathrm{Cu}-$ Poor and Cu-Rich CuInS 2 absorber layers, Phys. Rev. Appl. 11, 054052 (2019).

[16] A. Lomuscio, M. Melchiorre, and S. Siebentritt, Influence of stoichiometry and temperature on quasi Fermi level splitting of sulfide CIS absorber layers, in 2018 IEEE 7th World Conference on Photovoltaic Energy Conversion (WCPEC) (A Joint Conference of 45th IEEE PVSC, 28th PVSEC \& 34th EU PVSEC), Waikoloa Village, HI (IEEE, Piscataway, NJ, 2018).

[17] J. Binsma, L. Giling, and J. Bloem, Luminescence of $\mathrm{CuInS}_{2}$ : I. The broad band emission and its dependence on the defect chemistry, J. Lumin. 27, 35 (1982).

[18] H. Ueng and H. Hwang, The defect structure of $\mathrm{CuInS}_{2}$. Part I: Intrinsic defects, J. Phys. Chem. Solids 50, 1297 (1989).

[19] K. Töpper, J. Krauser, J. Bruns, R. Scheer, A. Weidinger, and D. Bräuning, Effects of post-deposition treatment on the PL spectra and the hydrogen content of $\mathrm{CuInS}_{2}$ absorber layers, Sol. Energy Mater. Sol. Cells 49, 383 (1997).

[20] J. Eberhardt, K. Schulz, H. Metzner, J. Cieslak, T. Hahn, U. Reislöhner, M. Gossla, F. Hudert, R. Goldhahn, and W. Witthuhn, Epitaxial and polycrystalline $\mathrm{CuInS}_{2}$ thin films: A comparison of opto-electronic properties, Thin Solid Films 515, 6147 (2007).

[21] I. Luck, J. Kneisel, K. Siemer, J. Bruns, R. Scheer, R. Klenk, N. Janke, and D. Bräunig, Influence of $\mathrm{Na}$ on the properties of $\mathrm{Cu}$-rich prepared $\mathrm{CuInS}_{2}$ thin films and the performance of corresponding $\mathrm{CuInS}_{2} / \mathrm{CdS} / \mathrm{ZnO}$ solar cells, Sol. Energy Mater. Sol. Cells 67, 151 (2001).

[22] S. Fiechter, Y. Tomm, M. Kanis, R. Scheer, and W. Kautek, On the homogeneity region, growth modes and optoelectronic properties of chalcopyrite-type $\mathrm{CuInS}_{2}$, Phys. Status Solidi 245, 1761 (2008).

[23] S. Siebentritt, N. Rega, A. Zajogin, and M. C. Lux-Steiner, Do we really need another PL study of CuInSe $\mathrm{Ce}_{2}$ ? Phys. Status Solidi 1, 2304 (2004).

[24] M. Weber, R. Scheer, H. Lewerenz, H. Jungblut, and U. Störkel, Microroughness and composition of cyanide-treated $\mathrm{CuInS}_{2}$, J. Electrochem. Soc. 149, G77 (2002).

[25] J. I. Pankove, Optical Processes in Semiconductors (Dover Publications Inc., New York, 1975). 
[26] T. Schmidt, K. Lischka, and W. Zulehner, Excitation-power dependence of the near-band-edge photoluminescence of semiconductors, Phys. Rev. B 45, 8989 (1992).

[27] See Supplemental Material at http://link.aps.org/supplemental/ 10.1103/PhysRevB.101.085119 for a comparison of band gap luminescence at different temperatures of the samples with $\mathrm{Cu} / \mathrm{In}$ of 1.82 and 1.00 ; equation used to fit the excitation intensity dependence PL flux; explanation of the attribution of the $\mathrm{C}$ line to $\mathrm{FB}$ transition; excitation intensity and temperature dependence spectra of DA2 at $10 \mathrm{~K}$; activation energies of the main admittance step measured on $\mathrm{Mo} / \mathrm{CuInS}_{2} / \mathrm{ZnOS} / \mathrm{AZO} / \mathrm{Al}$ stacks.

[28] C. Spindler, T. Galvani, L. Wirtz, G. Rey, and S. Siebentritt, Excitation-intensity dependence of shallow and deep-level photoluminescence transitions in semiconductors, J. Appl. Phys. 126, 175703 (2019).

[29] J. Binsma, L. Giling, and J. Bloem, Luminescence of $\mathrm{CuInS}_{2}$ : II. Exciton and near edge emission, J. Lumin. 27, 55 (1982).

[30] M. Yakushev, R. Martin, A. Mudryi, and A. Ivaniukovich, Excited states of the A free exciton in $\mathrm{CuInS}_{2}$, Appl. Phys. Lett. 92, 111908 (2008).

[31] M. Yakushev, A. Mudryi, I. Victorov, J. Krustok, and E. Mellikov, Energy of excitons in $\mathrm{CuInS}_{2}$ single crystals, Appl. Phys. Lett. 88, 011922 (2006).

[32] J. R. Haynes, Experimental Observation of the Excitonic Molecule, Phys. Rev. Lett. 17, 860 (1966).
[33] H. Atzmüller, F. Fröschl, and U. Schröder, Theory of excitons bound to neutral impurities in polar semiconductors, Phys. Rev. B. 19, 3118 (1979).

[34] R. R. Sharma and S. Rodriguez, Theory of excitons bound to ionized impurities in semiconductors, Phys. Rev. 153, 823 (1967).

[35] J. J. Hopfield, in Seventh International Conference on the Physics of Semiconductors (Dunod, Paris, 1964).

[36] P. Y. Yu and M. Cardona, Fundamentals of Semiconductors: Physics and Materials Properties (Springer, Berlin, 2010).

[37] L. Gütay, D. Regesch, J. K. Larsen, Y. Aida, V. Depredurand, A. Redinger, S. Caneva, S. Schorr, C. Stephan, J. Vidal, S. Botti, and S. Siebentritt, Feedback mechanism for the stability of the band gap of $\mathrm{CuInSe}_{2}$, Phys. Rev. B 86, 045216 (2012).

[38] W. Koschel and M. Bettini, Zone-centered phonons in AIBIIIS2 chalcopyrites, Phys. Status Solidi 72, 729, (1975).

[39] K. Wakita, H. Hirooka, S. Yasuda, F. Fujita, and N. Yamamoto, Resonant Raman scattering and luminescence in $\mathrm{CuInS}_{2}$ crystals, J. Appl. Phys. 83, 443 (1998).

[40] A. Alkauskas, M. D. McCluskey, and C. G. Van de Walle, Tutorial: Defects in semiconductors-Combining experiment and theory, J. Appl. Phys. 119, 181101 (2016).

[41] S. Siebentritt and U. Rau, Wide-Gap Chalcopyrites, Vol. 1 (Springer, Berlin, 2006). 